\title{
NEAR FIELD ASYMPTOTIC BEHAVIOR FOR THE POROUS MEDIUM EQUATION ON THE HALF-LINE
}

\author{
C. CORTÁZAR, F. QUIRÓS AND N. WOLANSKI
}

\begin{abstract}
Kamin and Vázquez proved in 1991 that solutions to the Cauchy-Dirichlet problem for the porous medium equation $u_{t}=\left(u^{m}\right)_{x x}$ on the half line with zero boundary data and nonnegative compactly supported integrable initial data behave for large times as a dipole type solution to the equation having the same first moment as the initial data, with an error which is $o\left(t^{-1 / m}\right)$. However, on sets of the form $0<x<g(t)$, with $g(t)=o\left(t^{1 /(2 m)}\right)$ as $t \rightarrow \infty$, in the so called near field, the dipole solution is $o\left(t^{-1 / m}\right)$, and their result does not give neither the right rate of decay of the solution, nor a nontrivial asymptotic profile. In this paper we will show that the error is $o\left(t^{-(2 m+1) /\left(2 m^{2}\right)}(1+x)^{1 / m}\right)$. This allows in particular to obtain a nontrivial asymptotic profile in the near field limit, which is a multiple of $x^{1 / m}$, thus improving in this scale the results of Kamin and Vázquez.
\end{abstract}

\section{INTRODUCTION}

This paper is concerned with the large time behavior of solutions to the porous medium equation (PME in what follows) in the half line,

$$
u_{t}=\left(u^{m}\right)_{x x}, \quad(x, t) \in \mathbb{R}_{+} \times \mathbb{R}_{+}, \quad u(x, 0)=u_{0}(x), \quad x \in \mathbb{R}_{+},
$$

with $m>1$, and nonnegative, and compactly supported integrable initial data. This problem, which models the flow of a fluid in a porous medium, has a unique weak solution; see [8]. The asymptotic behavior was first studied by Kamin and Vázquez in [7], and depends heavily on the fact that solutions to (1.1) preserve the first moment along the evolution, $\int_{0}^{\infty} x u(x, t) d x=$ constant for all $t>0$. Indeed, the behavior is given in terms of the so called dipole solution of the PME with first moment $M=\int_{0}^{\infty} x u_{0}(x) d x$,

$$
D_{M}(x, t)=t^{-\alpha} F_{M}(\xi), \quad \xi=x / t^{\beta}, \quad \alpha=\frac{1}{m}, \beta=\frac{1}{2 m} .
$$

Note that this solution has a self-similar structure, and that, due to the choice of the similarity exponents $\alpha$ and $\beta$, its first moment is constant in time. The precise result in [7] states that

$$
\lim _{t \rightarrow \infty} t^{\alpha} \sup _{x \in \mathbb{R}_{+}}\left|u(x, t)-D_{M}(x, t)\right|=0, \quad M=\int_{0}^{\infty} x u_{0}(x) d x .
$$

In order for $D_{M}$ to be a weak solution of the equation on the half-line, with $D_{M}(0, t)=0$, the profile $F_{M}$ has to solve, in a weak sense,

$$
\left(F_{M}^{m}\right)^{\prime \prime}(\xi)+\beta \xi F_{M}^{\prime}(\xi)+\alpha F_{M}(\xi)=0, \quad \xi \in \mathbb{R}_{+}, \quad F_{M}(0)=0,
$$

2010 Mathematics Subject Classification. 35B40, 35K65, 35R35.

Key words and phrases. Porous medium equation, half-line, asymptotic behavior, matched asymptotics.

C. Cortázar supported by FONDECYT grant 1150028 (Chile). F. Quirós supported by project MTM201453037-P (Spain). N. Wolanski supported by CONICET PIP625, Res. 960/12, ANPCyT PICT-2012-0153, UBACYT X117 and MathAmSud 13MATH03 (Argentina). 
while the condition on the value of the first moment imposes

$$
\int_{0}^{\infty} \xi F_{M}(\xi) d \xi=M
$$

A simple scaling argument shows that $F_{M}(\xi)=M^{1 / m} F_{1}\left(\xi / M^{(m-1) /(2 m)}\right)$. It turns out that there is a unique bounded profile corresponding to $M=1$, which is moreover explicit,

$$
F_{1}(\xi)=\xi^{\frac{1}{m}}\left(C_{m}-\kappa_{m} \xi^{\frac{m+1}{m}}\right)_{+}^{\frac{1}{m-1}}
$$

with constants $C_{m}$ and $\kappa_{m}$ given by

$$
\kappa_{m}=\frac{m-1}{2 m(m+1)}, \quad C_{m}=\left(\frac{\kappa_{m}^{\frac{2 m+1}{m+1}}}{\int_{0}^{1} s^{\frac{m+1}{m}}\left(1-s^{\frac{m+1}{m}}\right)^{\frac{1}{m-1}} d s}\right)^{\left(m^{2}-1\right) /\left(2 m^{2}\right)} ;
$$

see [2, 5, 6]. Note that $F_{1}$ has a compact support, $\left[0, \xi_{1}\right]$, where $\xi_{1}=\left(C_{m} / \kappa_{m}\right)^{m /(m+1)}$. Thus, $F_{M}$ has compact support $\left[0, \xi_{M}\right]$, with

$$
\xi_{M}=\xi_{1} M^{(m-1) /(2 m)} .
$$

Let us remark that $\lim _{t \rightarrow 0^{+}} \int_{0}^{\infty} D_{M}(x, t) \varphi(x) d x=M \varphi^{\prime}(0)$. In other words, the antisymmetric extension of $D_{M}, \bar{D}_{M}$, satisfies $\bar{D}_{M}(\cdot, t) \rightarrow-2 M \delta^{\prime}$, where $\delta^{\prime}$ is the distributional derivative of the delta function. This is called in physics an elementary dipole. Hence the name dipole solution of the PME for $D_{M}$.

Remarks. (a) The result in [7] states that solutions to the signed PME in the whole real line

$$
u_{t}=\left(|u|^{m-1} u\right)_{x x}, \quad(x, t) \in \mathbb{R} \times \mathbb{R}_{+}
$$

with an integrable and compactly supported initial data having zero mass and a nontrivial first moment $\int_{\mathbb{R}} x u(x, 0) d x=P$ converge to $\bar{D}_{P / 2}$, with an error which is $o\left(t^{-\alpha}\right)$. Solutions to (1.1) clearly fall within this framework, since they coincide with the restriction to the half line of the solution to (1.7) having as initial datum the antisymmetric extension of $u_{0}$.

(b) The proof in [7] uses that $v(x, t)=\int_{-\infty}^{x} u(y, t) d y$ is a solution to the $p$-laplacian evolution equation with $p=m+1$,

$$
v_{t}=\left(\left|v_{x}\right|^{m-1} v_{x}\right)_{x}, \quad(x, t) \in \mathbb{R} \times \mathbb{R}_{+} .
$$

Convergence for $u$ is deduced from the convergence of $v$ and its derivatives. In particular, $D_{M}=\partial_{x} B_{-2 M}$, where $B_{K}$ denotes the source type solution to (1.8), that has $K \delta$ as initial datum.

The limit (1.3) gives the first nontrivial term in the asymptotic expansion of $u$ for $x=O\left(t^{\beta}\right)$, in the so called far field limit. However, since $F_{M}(0)=0$, in the near field, $x=o\left(t^{\beta}\right)$, it only says that $u$ is $o\left(t^{-\alpha}\right)$. The aim of this paper is to improve the result of Kamin and Vázquez in the near field, giving a sharp decay rate, which is faster than that in the far field, and a nontrivial asymptotic profile, which turns out to be a multiple of $x^{1 / m}$. The precise result reads as follows.

Theorem 1.1. Let $u$ be the unique weak solution to (1.1). Then

$$
\lim _{t \rightarrow \infty} t^{\alpha+\frac{\beta}{m}} \sup _{x \in \mathbb{R}_{+}} \frac{\left|u(x, t)-D_{M}(x, t)\right|}{(1+x)^{\frac{1}{m}}}=0,
$$

where $D_{M}$ is the unique dipole solution to the PME with first moment $M=\int_{0}^{\infty} x u_{0}(x) d x$. 
In particular, on compact sets, $x \in[0, K], u$ is $O\left(t^{-\left(\alpha+\frac{\beta}{m}\right)}\right)$, and $t^{\alpha+\frac{\beta}{m}} u(x, t)$ converges to $M^{2 /(m+1)} C_{m}^{1 /(m-1)} x^{1 / m}$, while

$$
\sup _{0 \leq x \leq g(t)} u(x, t)=O\left(t^{\alpha+\frac{\beta}{m}} /(g(t))^{1 / m}\right)
$$

if $g(t) \rightarrow \infty, g(t)=o\left(t^{\beta}\right)$.

We already know that the result is true in the far-field scale, $\xi_{1} \leq x / t^{\beta} \leq \xi_{2}, 0<\xi_{1} \leq \xi_{2}<\infty$; see (1.3). If $x \geq g(t) t^{\beta}$, with $g(t) \rightarrow \infty$ as $t \rightarrow \infty$ (the very far field scale), formula (1.3) yields a better result. Nevertheless, since $D_{M}(x, t)=0$ for $x \geq \xi_{M} t^{\beta}$, it only says that the solution is $o\left(t^{-\alpha}\right)$ there. However, by using some asymptotic formulas from [4], Kamin and Vázquez prove that $s(t)=\sup \{x: u(x, t)>0\}$ satisfies

$$
s(t)=\xi_{M} t^{\beta}+o(1), \quad s(t) \geq \xi_{M} t^{\beta},
$$

which gives a complete characterization of the asymptotic behavior for $x>\xi_{M} t^{\beta}$. Hence, it only remains to check what happens in the near-field. This is done through a matching argument with the outer behavior, which is based on a clever choice of sub and supersolutions. Comparison is performed in sets of the form $0<x<\delta t^{\beta}$, for some small $\delta$. The ordering in the outer boundary comes from the outer behavior which was already known from the analysis in [7]. We devote Section 2 to obtain the upper limit and Section 3 to get the lower one.

A similar analysis for the linear heat equation has been recently performed in [3]. However, in that case linearity made things easier, since a representation formula for the solution in terms of the initial datum was available. That paper also considers a nonlocal version of the heat equation.

\section{Control from above}

The purpose of this section is to prove the "upper" part of (1.9). To this aim we will construct a supersolution $V$ approaching $D_{M}$ with the right rate as $t$ goes to infinity. We only need the function $V$ to be a supersolution in sets of the form

$$
A_{\delta, T}=\left\{(x, t), t \geq T, 0<x<\delta t^{\beta}\right\}
$$

for $T>0$ big and $\delta>0$ small. Our candidate is

$$
V(x, t)=k(t) t^{-\alpha} F_{M}\left(\frac{x+a}{t^{\beta}}\right), \quad a>0
$$

for some function $k$ satisfying $k(t) \searrow 1$ as $t \rightarrow \infty$. It will turn out that a good choice for $k$ is given by the solution to

$$
t k^{\prime}(t)=-\alpha\left(k^{m}(t)-k(t)\right), \quad t>T, \quad k(T)=k_{0}>1 .
$$

Note that $k(t)$ is well defined and that it is a monotone decreasing function of time.

We start by proving that $V$ is a supersolution to the PME in $A_{\delta, T}$ if $\delta$ is small and $T$ is big.

Lemma 2.1. Let $m>1$ and $M>0$. There exist values $\bar{\delta}>0$ and $\bar{T}>0$ depending only on $M$ and $m$ such that for all $a \in(0,1), T \geq \bar{T}$, and $k_{0}>1$ the function $V$ given by (2.1) (2.2) satisfies

$$
V_{t}-\left(V^{m}\right)_{x x} \geq 0 \quad \text { in } A_{\delta, T} \text { for all } \delta \in(0, \bar{\delta}) \text {. }
$$


Proof. Let $\xi=\frac{x+a}{t^{\beta}}$. Since $m \alpha+2 \beta=\alpha+1$, a straightforward computation combined with (1.4) shows that

$$
\left(V_{t}-\left(V^{m}\right)_{x x}\right)(x, t)=t^{-\alpha-1}\left(t k^{\prime}(t) F_{M}(\xi)+\left(k(t)-k^{m}(t)\right)\left(F_{M}^{m}\right)^{\prime \prime}(\xi)\right) .
$$

Thus, if we choose $k$ satisfying (2.2), we get

$$
\left(V_{t}-\left(V^{m}\right)_{x x}\right)(x, t)=t^{-\alpha-1}\left(k^{m}(t)-k(t)\right)\left(-\alpha F_{M}(\xi)-\left(F_{M}^{m}\right)^{\prime \prime}(\xi)\right), \quad x \in \mathbb{R}_{+}, t \geq T .
$$

Now we observe that there is a value $\bar{\xi} \in\left(0, \xi_{1}\right)$ such that $F_{1}^{\prime}(\xi)>0$ for $\xi \in(0, \bar{\xi})$. Therefore,

$$
F_{M}^{\prime}(\xi)>0, \quad \xi \in\left(0, \bar{\xi} M^{(m-1) /(2 m)}\right) .
$$

On the other hand, if we take $\bar{\delta}<\bar{\xi} M^{(m-1) /(2 m)} / 2$, and then $\bar{T}=(1 / \bar{\delta})^{1 / \beta}$, for any $\delta \in(0, \bar{\delta})$ and $T \geq \bar{T}$ we get

$$
0<\xi=\frac{x+a}{t^{\beta}} \leq 2 \bar{\delta}<\bar{\xi} M^{(m-1) /(2 m)}, \quad(x, t) \in A_{\delta, T} .
$$

Hence, $-\left(F_{M}^{m}\right)^{\prime \prime}(\xi)-\alpha F_{M}(\xi)=\beta \xi F_{M}^{\prime}(\xi)>0$ if $(x, t) \in A_{\delta, T}$, and the result follows, since $k(t)>1$ for all times.

We now arrive at the matching part of the result where, using the behavior in the far field scale, we obtain an upper bound in sets of the form $A_{\delta, T}$ for $\delta$ small and $T$ large.

Lemma 2.2. Let $u$ be the unique weak solution to (1.1), $M=\int_{0}^{\infty} x u_{0}(x) d x$, and $\bar{\delta}$ and $\bar{T}$ as in Lemma 2.1. For every $\varepsilon>0$ there exists a value $T_{\varepsilon} \geq \bar{T}$ such that for all $a \in(0,1)$, and $T \geq T_{\varepsilon}$ there is a value $k_{0} \geq 1$ such that the function $V$ given by (2.1)-(2.2) satisfies

$$
u(x, t) \leq\left(1+C_{\delta} \varepsilon\right) V(x, t), \quad(x, t) \in A_{\delta, T}, \quad C_{\delta}=1 / F_{M}(\delta), \quad \delta \in(0, \bar{\delta}) .
$$

Proof. Formula (1.3) implies that for every $\varepsilon>0$ there exists $T_{\varepsilon}$, which may be assumed to be larger than $\bar{T}$, such that

$$
t^{\alpha}\left|u(x, t)-D_{M}(x, t)\right| \leq \varepsilon, \quad x \in \mathbb{R}_{+}, t \geq T_{\varepsilon} .
$$

For any given $T \geq T_{\varepsilon}$ and some big enough $k_{0}>1$ to be determined below, we define $V$ by (2.1) (2.2).

Since $k(t)>1$, (2.3) implies that $D_{M}(x, t) \leq V(x, t)$ in $A_{\delta, \bar{T}}$, and hence in $A_{\delta, T}$. Therefore,

$$
t^{\alpha}(u(x, t)-V(x, t)) \leq t^{\alpha}\left|u(x, t)-D_{M}(x, t)\right|+t^{\alpha}\left(D_{M}(x, t)-V(x, t)\right) \leq \varepsilon, \quad(x, t) \in A_{\delta, T} .
$$

On the other hand, for $x=\delta t^{\beta}$ and $t \geq T$,

$$
t^{\alpha} V(x, t) \geq t^{\alpha} D_{M}(x, t)=F_{M}\left(\frac{x}{t^{\beta}}\right)=F_{M}(\delta),
$$

and we conclude that

$$
t^{\alpha}(u(x, t)-V(x, t)) \leq t^{\alpha} C_{\delta} \varepsilon V(x, t) .
$$

Thus,

$$
u(x, t) \leq \underbrace{\left(1+C_{\delta} \varepsilon\right) V(x, t)}_{W(x, t)} \quad \text { if } x=\delta t^{\beta}, t \geq T .
$$


Since solutions to (1.7) with integrable initial data are bounded for $t \geq \tau>0$, see [8], formula (1.3) implies that there is a constant $C_{0}>0$ such that $u(x, t) \leq C_{0} t^{-\alpha}$ for $t \geq \bar{T}$. Thus, using the monotonicity property (2.3), we get

$$
V(x, T)=k(T) T^{-\alpha} F_{M}\left(\frac{x+a}{T^{\beta}}\right) \geq k_{0} F_{M}\left(\frac{a}{T^{\beta}}\right) T^{-\alpha} \geq C_{0} T^{-\alpha} \geq u(x, T) \quad \text { for } 0<x<\delta T^{\beta}
$$

if $k_{0}>\max \left\{C_{0} / F_{M}\left(\frac{a}{T^{\beta}}\right), 1\right\}$. Therefore, with that choice of $k_{0}$,

$$
u(x, T) \leq V(x, T) \leq W(x, T) \text { if } 0<x<\delta T^{\beta} .
$$

We now observe that $W$ is a supersolution to the PME in $A_{\delta, T}$. Indeed, in that set we have $\left(V^{m}\right)_{x x}(x, t)=-k(t) t^{-(\alpha+1)}\left(\alpha F_{M}(\xi)+\beta \xi F_{M}^{\prime}(\xi)\right)<0$, and hence Lemma 2.2 implies that

$$
\begin{aligned}
W_{t}-\left(W^{m}\right)_{x x} & =\left(1+C_{\delta} \varepsilon\right) V_{t}-\left(1+C_{\delta} \varepsilon\right)^{m}\left(V^{m}\right)_{x x} \\
& =\left(1+C_{\delta} \varepsilon\right)\left(V_{t}-\left(V^{m}\right)_{x x}\right)-\left(\left(1+C_{\delta} \varepsilon\right)^{m}-\left(1+C_{\delta} \varepsilon\right)\right)\left(V^{m}\right)_{x x} \geq 0 .
\end{aligned}
$$

We finally notice that $W(0, t)>0$ for all $t>T$. Therefore, comparison yields (2.4).

The third ingredient, that we prove next, is that $V$ and $D_{M}$ are $\varepsilon$-close in sets of the form $A_{\delta, T}$ for large times, even when the difference is multiplied by $t^{\alpha+\frac{\beta}{m}} /(1+x)^{\frac{1}{m}}$, if the parameter $a$ in the definition of $V$ is $O\left(\varepsilon^{m}\right)$.

Lemma 2.3. Let $m>1, M>0$, and $\varepsilon>0$, and let $\bar{\delta}$ and $\bar{T}$ be as in Lemma 2.1. There exist values $\hat{\delta} \in(0, \bar{\delta}), \widehat{T} \geq \bar{T}$ independent of $\varepsilon$, and $a_{\varepsilon} \in(0,1]$, such that for all $\delta \in(0, \hat{\delta}), T \geq \widehat{T}$ and $a \in\left(0, a_{\varepsilon}\right)$ the function $V$ given by (2.1) -(2.2) satisfies

$$
\frac{t^{\alpha+\frac{\beta}{m}}}{(1+x)^{\frac{1}{m}}}\left|V(x, t)-D_{M}(x, t)\right|<\varepsilon \quad \text { in } A_{\delta, \widehat{T}_{\varepsilon}} \text { for some } \widehat{T}_{\varepsilon} \geq T \text {. }
$$

Proof. There holds that

$$
\begin{aligned}
\frac{t^{\alpha+\frac{\beta}{m}}}{(1+x)^{\frac{1}{m}}}\left|D_{M}(x, t)-V(x, t)\right|= & \underbrace{\frac{t^{\frac{\beta}{m}}}{(1+x)^{\frac{1}{m}}}\left|F_{M}\left(\frac{x+a}{t^{\beta}}\right)-F_{M}\left(\frac{x}{t^{\beta}}\right)\right|}_{\mathrm{I}} \\
& +\underbrace{\frac{t^{\frac{\beta}{m}}}{(1+x)^{\frac{1}{m}}} F_{M}\left(\frac{x+a}{t^{\beta}}\right)|k(t)-1|}_{\mathrm{II}} .
\end{aligned}
$$

In order to estimate I we notice that there exist constants $\hat{\xi} \in(0, \bar{\xi})$ and $K>0$ such that $\xi F_{1}^{\prime}(\xi) \leq K \xi^{\frac{1}{m}}$ for $\xi \in(0, \hat{\xi})$. Thus, if we take $\hat{\delta}<\hat{\xi} M^{(m-1) /(2 m)} / 2$, and then $\widehat{T}=(1 / \hat{\delta})^{1 / \beta}$, for any $\delta \in(0, \hat{\delta})$ and $T \geq \widehat{T}$, we get

$$
\begin{aligned}
\mathrm{I} & =\frac{t^{\frac{\beta}{m}}}{(1+x)^{\frac{1}{m}}} \int_{0}^{1} F_{M}^{\prime}\left(\frac{x+s a}{t^{\beta}}\right) \frac{a}{t^{\beta}} d s=\frac{t^{\frac{\beta}{m}}}{(1+x)^{\frac{1}{m}}} \int_{0}^{1} F_{M}^{\prime}\left(\frac{x+s a}{t^{\beta}}\right) \frac{x+s a}{t^{\beta}} \frac{a}{x+s a} d s \\
& \leq K \int_{0}^{1}(x+s a)^{\frac{1}{m}-1} a d s \leq m K a^{\frac{1}{m}} \quad \text { in } A_{\delta, T} .
\end{aligned}
$$

Therefore, I $<\varepsilon / 2$ if $a<a_{\varepsilon}:=\min \left\{(\varepsilon /(2 m K))^{m}, 1\right\}$. 
As for the other term, we will use that

$$
F_{M}(\xi) \leq C_{m}^{\frac{1}{m-1}} M^{\frac{m+1}{2 m^{2}}} \xi^{\frac{1}{m}}, \quad \xi \in \mathbb{R}_{+}
$$

see formula (1.4). Therefore, taking into account that $a<1$, we obtain

$$
\mathrm{II} \leq C_{m}^{\frac{1}{m-1}} M^{\frac{m+1}{2 m^{2}}}\left(\frac{x+a}{x+1}\right)^{\frac{1}{m}}|k(t)-1| \leq C_{m}^{\frac{1}{m-1}} M^{\frac{m+1}{2 m^{2}}}|k(t)-1|<\varepsilon / 2
$$

if $t \geq \widehat{T}_{\varepsilon}$ for some $\widehat{T}_{\varepsilon} \geq T$, since $k(t) \rightarrow 1$ as $t \rightarrow \infty$.

We finally arrive at the main result of this section, the upper limit.

Proposition 2.1. Let $u$ be the unique weak solution to (1.1), and $D_{M}$ be the unique dipole solution to the PME with first moment $M=\int_{0}^{\infty} x u_{0}(x) d x$. If $\hat{\delta}$ is the constant given by Lemma 2.3, then, for all $\delta \in(0, \hat{\delta})$,

$$
\limsup _{t \rightarrow \infty} t^{\alpha+\frac{\beta}{m}} \sup _{0<x<\delta t^{\beta}} \frac{\left(u(x, t)-D_{M}(x, t)\right)}{(1+x)^{\frac{1}{m}}} \leq 0 .
$$

Proof. Given $\varepsilon>0$, let $T_{\varepsilon}$ as in Lemma 2.2, and $\widehat{T}$ and $a_{\varepsilon}$ as in Lemma 2.3. We take $T \geq$ $\max \left\{T_{\varepsilon}, \widehat{T}\right\}$ and $a \in\left(0, a_{\varepsilon}\right)$, and then $k_{0}>1$ large so that the function $V$ defined by (2.1)-(2.2) satisfies (2.4) and (2.6) for any given $\delta \in(0, \hat{\delta})$ for some large $\widehat{T}_{\varepsilon} \geq T$.

On the other hand, since $k(t) \rightarrow 1$ as $t \rightarrow \infty$ and $a \in(0,1)$, using (2.7) we get

$$
\frac{t^{\alpha+\frac{\beta}{m}} V(x, t)}{(1+x)^{\frac{1}{m}}}=\frac{k(t) t^{\frac{\beta}{m}}}{(1+x)^{\frac{1}{m}}} F_{M}\left(\frac{x+a}{t^{\beta}}\right) \leq 2 C_{m}^{\frac{1}{m-1}} M^{\frac{m+1}{2 m^{2}}}\left(\frac{x+a}{x+1}\right)^{\frac{1}{m}} \leq 2 C_{m}^{\frac{1}{m-1}} M^{\frac{m+1}{2 m^{2}}}
$$

for all large enough times.

Combining all the estimates mentioned above we finally get, for $0<x<\delta t^{\beta}$ and all large enough times,

$$
\frac{t^{\alpha+\frac{\beta}{m}}}{(1+x)^{\frac{1}{m}}}\left(u(x, t)-D_{M}(x, t)\right) \leq C_{\delta} \varepsilon \frac{t^{\alpha+\frac{\beta}{m}} V(x, t)}{(1+x)^{\frac{1}{m}}}+\varepsilon \leq\left(C_{\delta} 2 C_{m}^{\frac{1}{m-1}} M^{\frac{m+1}{2 m^{2}}}+1\right) \varepsilon .
$$

\section{Control from Below}

We will now deal with the "lower" part of (1.9). The proof is quite similar to that of the "upper" part. However, in this case, subsolutions are only obtained in sets of the form

$$
A_{a, \delta, T}=\left\{(x, t): a<x<\delta t^{\beta}, t \geq T\right\},
$$

and the points $x \in(0, a)$ have to be treated separately.

The subsolution approaching $D_{M}$ with the right rate as $t$ goes to infinity will have the form

$$
v(x, t)=c(t) t^{-\alpha} F_{M}\left(\frac{x-a}{t^{\beta}}\right), \quad a>0,
$$

where $c$ is the solution to the Initial Value Problem

$$
t c^{\prime}(t)=\alpha\left(c(t)-c^{m}(t)\right), \quad t>T, \quad c(T)=c_{0} \in(0,1) .
$$


The function $c$ is well defined for $t \geq T$. It is monotone increasing and $c(t) \nearrow 1$ as $t \rightarrow \infty$, as desired.

We start by proving that $v$ is a subsolution to the PME in $A_{a, \delta, T}$ if $\delta$ is small and $T$ is big, no matter the value of $a \in(0,1)$.

Lemma 3.1. Let $m>1$ and $M>0$, and let $\bar{\delta}>0$ be as in Lemma 2.1. For all $a \in(0,1)$, $T>0$, and $c_{0} \in(0,1)$ the function $v$ given by (3.1)-(3.2) satisfies

$$
v_{t}-\left(v^{m}\right)_{x x} \leq 0 \quad \text { in } A_{a, \delta, T} \text { for all } \delta \in(0, \bar{\delta}) \text {. }
$$

Proof. Let $\xi=\frac{x-a}{t^{\beta}}<\frac{x}{t^{\beta}}$. A computation analogous to the one we did in the proof of Lemma 2.1 shows that

$$
\left(v_{t}-\left(v^{m}\right)_{x x}\right)(x, t)=-t^{-\alpha-1}\left(c(t)-c^{m}(t)\right) \beta \xi F_{M}^{\prime}(\xi) .
$$

But,

$$
0<\xi=\frac{x-a}{t^{\beta}}<\frac{x}{t^{\beta}}<\delta<\bar{\delta}<\bar{\xi} M^{(m-1) /(2 m)}, \quad(x, t) \in A_{a, \delta, T},
$$

and hence the result follows from (2.3), since $c(t)<1$ for all times.

The matching with the outer behavior will require to know that $u$ is positive in some set $A_{\delta, T}$. This is what we prove next.

Lemma 3.2. Let $u$ be the unique weak solution to (1.1), $M=\int_{0}^{\infty} x u_{0}(x) d x$. Given $\delta \in\left(0, \xi_{M}\right)$, there exists a time $T_{\delta}$ such that $u(x, t)>0$ in $A_{\delta, T_{\delta}}$.

Proof. Since $\delta<\xi_{M}$, the convergence result (1.3) implies that there is a time $t_{\delta}$ such that $u(x, t) \geq K t^{-\alpha}$ for some $K>0$ if $x \in\left((\delta / 2) t^{\beta}, \delta t^{\beta}\right), t \geq t_{\delta}$.

We now use that non-negative solutions to (1.1) have the so-called retention property: if $u(x, \bar{t})>0$, then $u(x, t)>0$ for all $t \geq \bar{t}$. This can be proved in several ways, for instance, using that the application $t \mapsto t^{1 /(m-1)} u(x, t)$ is non-decreasing. This monotonicity property follows easily from the estimate $u_{t} \geq-u /((m-1) t)$, which is proved using comparison arguments; see, for instance, [8]. Hence, $u(x, t)>0$ for $x \in\left((\delta / 2) t_{\delta}^{\beta}, \delta t^{\beta}\right), t \geq t_{\delta}$.

It only remains to prove that $u\left(x, T_{\delta}\right)>0$ if $x \in\left(0,(\delta / 2) T_{\delta}^{\beta}\right)$ for some large enough $T_{\delta} \geq t_{\delta}$, since the result will then follow from the retention property. The positivity in this fixed interval is achieved by comparison with a suitable translate of a source type solution of the PME,

$$
B(x, t ; C)=t^{-\frac{1}{m+1}}\left(C-\kappa_{m}|\xi|^{2}\right)_{+}^{\frac{1}{m-1}}, \quad \xi=x / t^{\frac{1}{m+1}}, \quad C>0,
$$

where the constant $\kappa_{m}$ is as in (1.6). Such solutions are due to Zel'dovič and Kompaneets [9] and Barenblatt [1]. Indeed, take $x_{0} \in\left((\delta / 2) t_{\delta}^{\beta}, \delta t_{\delta}^{\beta}\right)$. It is easy to check that if $C>0$ is small enough, then

$$
B\left(x-x_{0}, t_{\delta} ; C\right)=0 \quad \text { if } x \notin\left((\delta / 2) t_{\delta}^{\beta}, \delta t_{\delta}^{\beta}\right), \quad \sup _{(\delta / 2) t_{\delta}^{\beta} \leq x \leq \delta t_{\delta}^{\beta}} B\left(x-x_{0}, t_{\delta} ; C\right) \leq K t_{\delta}^{-\alpha} .
$$

Moreover, $B\left(x-x_{0}, t ; C\right)$ is a solution to (1.1) until it touches the boundary $x=0$. This will happen in a finite time $T_{\delta} \geq t_{\delta}$. Then, comparison yields that $u(x, t) \geq B\left(x-x_{0}, t ; C\right)$ for all $t \in\left[t_{\delta}, T_{\delta}\right]$, and hence the required positivity.

We now perform the matching with the outer behavior in order to obtain the control from below. 
Lemma 3.3. Let $u$ be the unique weak solution to (1.1), $M=\int_{0}^{\infty} x u_{0}(x) d x$, and $\bar{\delta}$ as in Lemma 2.1. Given $\varepsilon>0, a \in(0,1)$, and $\delta \in(0, \bar{\delta})$, there is a time $T_{\varepsilon, a, \delta}>0$ such that for all $T \geq T_{\varepsilon, a, \delta}$ there is a value $c_{0} \in(0,1)$ such that the function $v$ given by (3.1) -(3.2) satisfies

$$
u(x, t) \geq\left(1-C_{\delta} \varepsilon\right) v(x, t), \quad(x, t) \in A_{a, \delta, T}, \quad C_{\delta}=1 / F_{M}(\delta) .
$$

Proof. Let $\delta \in(0, \bar{\delta})$ and $a \in(0,1)$. Note that $\bar{\delta}<\xi_{M}$. The convergence result (1.3) implies that, given $\varepsilon>0$, there exists a value $T_{a, \varepsilon, \delta} \geq \max \left\{T_{\bar{\delta}},(a / \delta)^{1 / \beta}\right\}$ such that (2.5) holds with $T_{\varepsilon}=T_{\varepsilon, a, \delta}$.

Let $T \geq T_{\varepsilon, a, \delta}$. We know from Lemma 3.2 that there is a constant $\kappa=\kappa(a, \delta, T)$ such that $u(x, T) \geq \kappa$ if $a<x<\delta T^{\beta}$. Take now $c_{0} \in(0,1)$ small so that $c_{0} T^{-\alpha} F_{M}(\delta) \leq \kappa$. With this choice of $T$ and $c_{0}$, we define $v$ by (3.1)-(3.2). Using the monotonicity property (2.3), we get

$$
u(x, T) \geq c_{0} T^{-\alpha} F_{M}\left(\frac{x-a}{T}\right)=v(x, T), \quad a<x<\delta T^{\beta} .
$$

On the other hand, since $T \geq T_{\varepsilon, a, \delta}$, the convergence result (1.3) together with the selfsimilar form of $D_{M}$, formula (1.2) yield

$$
u(x, t) \geq-\varepsilon t^{-\alpha}+D_{M}(x, t)=\left(1-F_{M}(\delta)^{-1} \varepsilon\right) D_{M}(x, t), \quad x=\delta t^{\beta}, t \geq T .
$$

We notice now that $c(t)<1$. Therefore, (2.3) implies that $D_{M} \geq v$ in $A_{\delta, T}$, and we conclude that, for $\varepsilon<F_{M}(\delta)$ and $C_{\delta}=1 / F_{M}(\delta)$,

$$
u(x, t) \geq \underbrace{\left(1-C_{\delta} \varepsilon\right) v(x, t)}_{w(x, t)}, \quad x=\delta t^{\beta}, t \geq T .
$$

We now observe that $w$ is a subsolution to the PME in $A_{a, \delta, T}$. Indeed, in that set we have $\left(v^{m}\right)_{x x}(x, t)=-c(t) t^{-(\alpha+1)}\left(\alpha F_{M}(\xi)+\beta \xi F_{M}^{\prime}(\xi)\right)<0$, and hence Lemma 3.1 implies that

$$
w_{t}-\left(w^{m}\right)_{x x}=\left(1-C_{\delta} \varepsilon\right)\left(v_{t}-\left(v^{m}\right)_{x x}\right)-\left(\left(1-C_{\delta} \varepsilon\right)^{m}-\left(1-C_{\delta} \varepsilon\right)\right)\left(v^{m}\right)_{x x} \leq 0 \text { in } A_{a, \delta, T} \text {. }
$$

We finally notice that $w(a, t)=0$ for all $t>T$. Therefore, a comparison argument allows to conclude that (3.4) holds.

The next step is to control the difference between $v$ and $D_{M}$ for large times.

Lemma 3.4. Given $m>1, M>0$, and $\varepsilon>0$, let $\hat{\delta}$ and $a_{\varepsilon} \in(0,1]$ be as in Lemma 2.3. Then, for all $\delta \in(0, \hat{\delta}), T>0$, and $a \in\left(0, a_{\varepsilon}\right)$, the function $v$ given by (3.1)-(3.2) satisfies

$$
\frac{t^{\alpha+\frac{\beta}{m}}}{(1+x)^{\frac{1}{m}}}\left|D_{M}(x, t)-v(x, t)\right|<\varepsilon \quad \text { in } A_{a, \delta, \widetilde{T}_{\varepsilon}} \text { for some } \widetilde{T}_{\varepsilon} \geq T \text {. }
$$

Proof. Let $x \in\left(a, \delta t^{\beta}\right)$ with $\delta<\hat{\delta}$. Arguing as in the proof of Lemma 2.3 we get

$$
\begin{gathered}
\frac{t^{\alpha+\frac{\beta}{m}}}{(1+x)^{\frac{1}{m}}}\left|t^{-\alpha} F_{M}\left(\frac{x-a}{t^{\beta}}\right)-D_{M}(x, t)\right| \leq \frac{t^{\frac{\beta}{m}}}{(1+x)^{\frac{1}{m}}} \int_{0}^{1} F_{M}^{\prime}\left(\frac{x-s a}{t^{\beta}}\right) \frac{x-s a}{t^{\beta}} \frac{a}{x-s a} d s \\
\leq K \int_{0}^{1}(x-s a)^{\frac{1}{m}-1} a d s \leq m K a^{\frac{1}{m}}<\frac{\varepsilon}{2}
\end{gathered}
$$

if $a<a_{\varepsilon}:=\min \left\{(\varepsilon /(2 m K))^{m}, 1\right\}$. 
On the other hand, using (2.7),

$$
\begin{aligned}
& \frac{t^{\alpha+\frac{\beta}{m}}}{(1+x)^{\frac{1}{m}}}\left|t^{-\alpha} F_{M}\left(\frac{x-a}{t^{\beta}}\right)-v(x, t)\right|=\frac{t^{\frac{\beta}{m}}}{(1+x)^{\frac{1}{m}}} F_{M}\left(\frac{x-a}{t^{\beta}}\right)|1-c(t)| \\
& \quad \leq C_{m}^{\frac{1}{m-1}} M^{\frac{m+1}{2 m^{2}}}\left(\frac{x-a}{1+x}\right)^{\frac{1}{m}}|1-c(t)| \leq C_{m}^{\frac{1}{m-1}} M^{\frac{m+1}{2 m^{2}}}|1-c(t)|<\frac{\varepsilon}{2}
\end{aligned}
$$

if $t \geq \widetilde{T}_{\varepsilon}$ for some large enough $\widetilde{T}_{\varepsilon} \geq T$, since $c(t) \rightarrow 1$ as $t \rightarrow \infty$.

The combination of the above two estimates yields the result.

We now have all the tools we need to prove the lower limit. Here a difference arises with respect to the upper limit: we will have to treat separately the limit in sets of the form $(0, a)$ with $a$ small. This is done using that both $u$ and $D_{M}$ are small in that set for large times.

Proposition 3.1. Let $u$ be the unique weak solution to (1.1), and $D_{M}$ be the unique dipole solution to the PME with first moment $M=\int_{0}^{\infty} x u_{0}(x) d x$. If $\hat{\delta}$ is the constant given by Lemma [2.3, then, for all $\delta \in(0, \hat{\delta})$,

$$
\liminf _{t \rightarrow \infty} t^{\alpha+\frac{\beta}{m}} \sup _{0<x<\delta t^{\beta}} \frac{\left(u(x, t)-D_{M}(x, t)\right)}{(1+x)^{\frac{1}{m}}} \geq 0 .
$$

Proof. Given $\delta \in(0, \hat{\delta})$ and $\varepsilon \in\left(0, F_{M}(\delta)\right)$, we choose a small value $a \in\left(0, a_{\varepsilon}\right)$, with $a_{\varepsilon} \in(0,1]$ as in Lemma 2.3. We will precise how small it has to be later on. We take $T \geq \max \left\{T_{\varepsilon}, T_{\varepsilon, a, \delta}\right\}$, with $T_{\varepsilon}$ as in Lemma 2.2, and $T_{\varepsilon, a, \delta}$ as in Lemma 3.3. and then $c_{0} \in(0,1)$ small enough so that the function $v$ defined by (3.1)-(3.2) satisfies (3.4) and (3.5).

By Lemma 2.2. we know that there is a value $k_{0} \geq 1$ such that the function $V$ defined by (2.1)-(2.2) satisfies (2.4). Besides, since $k(t) \rightarrow 1$ as $t \rightarrow \infty$, there exists a time $\check{T} \geq T$ such that $k(t) \leq 2$ for all $t \geq \check{T}$. Therefore, since $C_{\delta} \varepsilon<1$, using (2.7) we get

$$
\begin{aligned}
\frac{t^{\alpha+\frac{\beta}{m}} u(x, t)}{(1+x)^{\frac{1}{m}}} & \leq\left(1+C_{\delta} \varepsilon\right) \frac{t^{\alpha+\frac{\beta}{m}} V(x, t)}{(1+x)^{\frac{1}{m}}} \\
& \leq 2\left(1+C_{\delta} \varepsilon\right) \frac{t^{\frac{\beta}{m}}}{(1+x)^{\frac{1}{m}}} F_{M}\left(\frac{x+a}{t^{\beta}}\right) \leq 4 C_{m}^{\frac{1}{m-1}} M^{\frac{m+1}{2 m^{2}}}(2 a)^{\frac{1}{m}}<\frac{\varepsilon}{2},
\end{aligned}
$$

if $a$ is small enough. On the other hand, using again (2.7),

$$
\frac{t^{\alpha+\frac{\beta}{m}} D_{M}(x, t)}{(1+x)^{\frac{1}{m}}}=\frac{t^{\frac{\beta}{m}}}{(1+x)^{\frac{1}{m}}} F_{M}\left(\frac{x}{t^{\beta}}\right) \leq C_{m^{\frac{1}{m-1}}}^{\frac{m+1}{2 m^{2}}} a^{\frac{1}{m}}<\frac{\varepsilon}{2} .
$$

We conclude that

$$
t^{\alpha+\frac{\beta}{m}} \sup _{0<x<a} \frac{\left|u(x, t)-D_{M}(x, t)\right|}{(1+x)^{\frac{1}{m}}} \leq \varepsilon \quad \text { if } t \text { is large enough. }
$$

We now consider the set $a<x<\delta t^{\beta}$. Since $c(t) \rightarrow 1$ as $t \rightarrow \infty$, using (2.7) we get

$$
\frac{t^{\alpha+\frac{\beta}{m}} v(x, t)}{(1+x)^{\frac{1}{m}}}=\frac{c(t) t^{\frac{\beta}{m}}}{(1+x)^{\frac{1}{m}}} F_{M}\left(\frac{x-a}{t^{\beta}}\right) \leq 2 C_{m}^{\frac{1}{m-1}} M^{\frac{m+1}{2 m^{2}}}\left(\frac{x-a}{x+1}\right)^{\frac{1}{m}} \leq 2 C_{m}^{\frac{1}{m-1}} M^{\frac{m+1}{2 m^{2}}}
$$


for all large enough times. Combining this estimate with (3.4) and (3.5), we finally get, for $a<x<\delta t^{\beta}$ and all large enough times,

$$
\frac{t^{\alpha+\frac{\beta}{m}}}{(1+x)^{\frac{1}{m}}}\left(u(x, t)-D_{M}(x, t)\right) \geq-C_{\delta} \frac{t^{\alpha+\frac{\beta}{m}} v(x, t)}{(1+x)^{\frac{1}{m}}}-\varepsilon \geq-\left(C_{\delta} 2 C_{m}^{\frac{1}{m-1}} M^{\frac{m+1}{2 m^{2}}}+1\right) \varepsilon .
$$

\section{REFERENCES}

[1] Barenblatt, G. I. On some unsteady motions of a liquid and gas in a porous medium. (Russian) Akad. Nauk SSSR. Prikl. Mat. Meh. 16 (1952), no. 1, 67-78.

[2] Barenblatt, G.I.; Zel'dovic, Ya. B. On dipole solutions in problems of non-stationary filtration of gas under polytropic regime. (Russian) Prikl. Mat. Mekh. 21 (1957), no. 5, 718-720.

[3] Cortázar, C.; Elgueta, M.; Quirós, F.; Wolanski, N. Asymptotic behavior for a nonlocal diffusion equation on the half line. Discrete Contin. Dyn. Syst. 35 (2015), no. 4, 1391-1407.

[4] Esteban, J. R.; Vázquez, J. L. Homogeneous diffusion in $R$ with power-like nonlinear diffusivity. Arch. Rational Mech. Anal. 103 (1988), no. 1, 39-80.

[5] Gilding, B. H.; Peletier, L. A. On a class of similarity solutions of the porous media equation. J. Math. Anal. Appl. 55 (1976), no. 2, 351-364.

[6] Gilding, B. H.; Peletier, L. A. On a class of similarity solutions of the porous media equation. II. J. Math. Anal. Appl. 57 (1977), no. 3, 522-538.

[7] Kamin, S.; Vázquez, J. L. Asymptotic behaviour of solutions of the porous medium equation with changing sign. SIAM J. Math. Anal. 22 (1991), no. 1, 34-45.

[8] Vázquez, J. L. "The porous medium equation. Mathematical theory". Oxford Mathematical Monographs. The Clarendon Press, Oxford University Press, Oxford, 2007. ISBN: 978-0-19-856903-9.

[9] Zel'dovič, Ya. B.; Kompaneets, A.S. On the theory of propagation of heat with the heat conductivity depending upon the temperature. Collection in honor of the seventieth birthday of academician A. F. Ioffe, pp. 61-71. Izdat. Akad. Nauk SSSR, Moscow, 1950.

\section{CARMen Cortázar}

Departamento de Matemática, Pontificia Universidad Católica de Chile

Santiago, Chile.

E-mail address: ccortaza@mat.puc.cl

FERNANDO Quirós

Departamento de Matemáticas, Universidad Autónoma de Madrid 28049-MADRID, SPAIN.

E-mail address: fernando.quiros@uam.es

Noemi Wolanski

Departamento de Matemática, FCEyn, UBA,

AND IMAS, CONICET,

Ciudad Universitaria, Pab. I,

(1428) Buenos Aires, Argentina.

E-mail address: wolanski@dm.uba.ar 